\title{
The role of lipid composition on the interaction between a tryptophan-rich protein and model bacterial membranes
}

Article

Accepted Version

Sanders, M. R., Clifton, L. A., Frazier, R. A. and Green, R. J. (2016) The role of lipid composition on the interaction between a tryptophan-rich protein and model bacterial membranes. Langmuir, 32 (8). pp. 2050-2057. ISSN 0743-7463 doi: https://doi.org/10.1021/acs.langmuir.5b04628 Available at https://centaur.reading.ac.uk/52906/

It is advisable to refer to the publisher's version if you intend to cite from the work. See Guidance on citing.

To link to this article DOI: http://dx.doi.org/10.1021/acs.langmuir.5b04628

Publisher: American Chemical Society

All outputs in CentAUR are protected by Intellectual Property Rights law, including copyright law. Copyright and IPR is retained by the creators or other copyright holders. Terms and conditions for use of this material are defined in the End User Agreement.

www.reading.ac.uk/centaur 
Central Archive at the University of Reading

Reading's research outputs online 


\section{The role of lipid composition on the interaction}

\section{between a tryptophan-rich protein and model}

\section{bacterial membranes}

Michael R. Sanders ${ }^{a}$, Luke A. Clifton ${ }^{b}$, Richard A. Frazier*a and Rebecca J. Green*a

*Corresponding Authors

aSchool of Pharmacy and Department of Food and Nutritional Sciences, University of Reading, PO Box 226, Whiteknights, Reading, Berkshire, RG6 6AP.

${ }^{b}$ ISIS Pulsed Neutron and Muon Source, Science and technology Facilities Council, Rutherford Appleton Laboratory, Harwell Oxford Campus, Didcot, Oxfordshire, OX11 0QX

KEYWORDS: Lipid monolayer, biological membrane mimic, bacterial membrane, plasma membrane, antimicrobial protein, puroindoline, FTIR spectroscopy, surface pressure, Brewster angle microscopy

\section{ABSTRACT}

The interaction between tryptophan-rich puroindoline proteins and model bacterial membranes at the air-liquid interface has been investigated by FTIR spectroscopy, surface pressure measurements and Brewster angle microscopy. The role of different lipid constituents on the 
interactions between lipid membrane and protein was studied using wild type (Pin-b) and mutant (Trp44 to Arg44 mutant, Pin-bs) puroindoline proteins. The results show differences in the lipid selectivity of the two proteins in terms of preferential binding to specific lipid head groups in mixed lipid systems. Pin-b wild type was able to penetrate mixed layers of phosphatidylethanolamine (PE) and phosphatidylglycerol (PG) head groups more deeply compared to the mutant Pin-bs. Increasing saturation of the lipid tails increased penetration and adsorption of Pin-b wild type, but again the response of the mutant form differed. The results provide insight as to the role of membrane architecture, lipid composition and fluidity, on antimicrobial activity of proteins. Data show distinct differences in the lipid binding behavior of Pin-b as a result of a single residue mutation, highlighting the importance of hydrophobic and charged amino acids in antimicrobial protein and peptide activity.

\section{INTRODUCTION}

Antimicrobial proteins and peptides bind to biological membranes via interactions with lipids and thus the lipid composition controls the membrane binding activity of the protein. This allows proteins to show selectivity towards particular cell types, a phenomenon that has been shown to depend on the amino acid composition of the membrane-binding region of the protein ${ }^{1}$ or peptide $^{2}$. In our previous studies we have used simple 1,2-dipalmitoyl-sn-glycero-3phosphocholine (DPPC) and 1,2-dipalmitoyl-sn-glycero-3-phospho-(1'-rac-glycerol) (DPPG) monolayers and observed that a single point mutation within the tryptophan-rich loop of puroindoline-b (Pin-b) reduces the ability of the protein to discriminate between these lipid surfaces. ${ }^{3}$ Lipid interacting proteins and peptides, such as puroindolines, can be seen as 
peripheral membrane proteins and like other membrane proteins the activity of these proteins have been shown to be greatly affected by the lipid architecture of the biological membrane. ${ }^{4}$

There are two primary ways that lipids affect protein structure and function. The first is that protein function is influenced by specific protein-lipid interactions that depend on the individual chemical and structural characteristics of the lipids ${ }^{5-7}$, e.g. head group, alkyl chain length and degree of unsaturation. The second is that protein function is influenced by self-association properties that result from collective properties ${ }^{8-10}$, e.g. fluidity, shape and packing properties. In the case of plant defence proteins, it is the properties of the plasma membrane of pathogenic species that decides the interaction and therefore the antimicrobial activity. ${ }^{11}$

All species of bacteria can be split into Gram positive and Gram-negative bacteria, for which the membrane structure and composition is significantly different. Gram-negative bacteria membranes are made up of an outer membrane and an inner plasma membrane each composed of different lipids. In contrast Gram-positive bacteria only have an inner plasma membrane. Additionally the phospholipid head group composition of the plasma membrane is not constant between species of Gram positive and Gram-negative bacteria can vary significantly. ${ }^{4}$ There are three phospholipid head groups that dominate the overall composition of these systems; the anionic component is dominated by phosphatidylglycerol (PG) and cardiolipin whereas the zwitterionic component is provided by the phosphatidylethanolamine (PE). ${ }^{12}$ Head group composition is not the only characteristic that changes from species to species; lipid tail chain length and saturation vary and bacterial cells are native to a broad range of environments. ${ }^{13}$ Therefore it is advantageous for bacterial species to be able to control the fluidity and thickness of plasma membrane in vivo via differences in lipid tail composition. ${ }^{4}$ 
Puroindolines (Pins) are cysteine-rich basic proteins isolated from wheat. The wild type isoforms, Pin-a and Pin-b both possess a hydrophobic tryptophan rich domain (TRD) within the amino acid sequence. ${ }^{4}$ Experiments have shown that this domain plays a role in conferring a soft endosperm texture, since hard wheat varieties are found to have the Pin protein missing or mutations within the tryptophan domain (Figure 1). It is believed that differences in endosperm texture are due to the starch binding of these proteins and are lipid-mediated. ${ }^{15-16}$ The Pins have been shown to be antimicrobial and to act against plant pathogens. The biological function of these proteins appears to be linked to their lipid binding abilities..$^{15,17-18}$

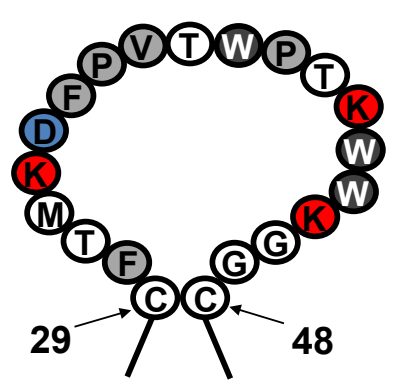

Pin-b

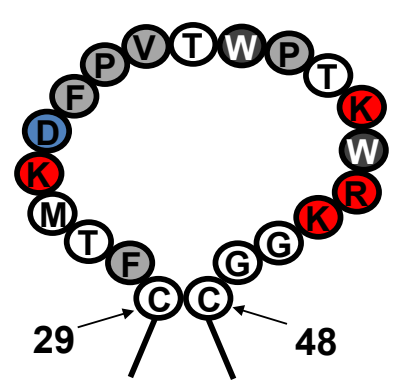

Pin-bs

Figure 1. The amino acid sequences of the tryptophan-rich domain of Pin-b wild type (left) and the Trp 44-Arg-44 mutant form, Pin-bs (right). The amino acids are coloured to differentiate characteristics: hydrophobic (grey), negative charged (blue) and positively charged (red).

Here we investigate for the first time how different lipid characteristics, through varying the complexity of the lipid system of study, affect the puroindoline binding to lipid monolayers. In addition, the role of tryptophan in protein-lipid binding is studied by comparison of binding behavior of the Pin-b wild type and Trp 44-Arg 44 mutant form (Pin-bs). Brewster angle microscopy (BAM), surface pressure measurements and external reflection-Fourier transform 
infrared (ER-FTIR) spectroscopy are utilized to in parallel experiments to provide information relating to lipid penetration of protein into the lipid layer surface and protein adsorption at the lipid surface that enable the mode of interaction to be characterized.

\section{MATERIALS AND METHODS}

Materials. All phospholipids and total lipid extracts were obtained as powders from Avanti Polar Lipids (Madison, WI, USA) without further purification. Phospholipid powders were suspended in HPLC chloroform (Sigma-Aldrich) with exception to lipids containing the phospholipid head group PE, which was suspended in 1:3 HPLC methanol: HPLC chloroform (Sigma-Aldrich). All stock solutions were made to a concentration of $1 \mathrm{mg} \mathrm{ml}^{-1}$. Mixed lipid solutions were prepared from single lipid solutions using a Hamilton glass microsyringe to ensure accuracy. The binary phospholipid systems tested were proportionally mixed with saturated DPPG in the following series; $20 \%, 40 \%, 60 \%, 80 \%$ and $90 \%$ DPPE at a total lipid concentration of $1 \mathrm{mg} \mathrm{ml}^{-1}$. Buffers were made from a combination of monosodium phosphate and disodium phosphate (dihydrate) to make buffer strength of $20 \mathrm{mM}$ with a $\mathrm{pH}$ or $\mathrm{pD} 7.0$, where either ultra high quality (UHQ) water $\left(18.2 \mathrm{M} \Omega\right.$ ) or $\mathrm{D}_{2} \mathrm{O}$ was used as the appropriate solvent. The $\mathrm{pH}$ was checked using a $\mathrm{pH}$ meter and adjusted using the buffer salts accordingly. UHQ water was obtained from an ELGA water purifier and 99.9\% D2O was obtained from Sigma-Aldrich Pin-b and Pin-bs were purified from Claire and Soissons wheat varieties respectively using Triton $\mathrm{X}-114$ phase partioning and chromatography techniques as described elsewhere. ${ }^{19-20}$ 
Surface pressure measurements. Surface pressure measurements of protein-lipid interactions were carried out on a Langmuir Teflon trough (model 611, Nima technology Ltd, Coventry, UK) with all the lipid monolayers prepared as previously described. ${ }^{21}$ Lipid monolayers were compressed at a barrier speed of $20 \mathrm{~cm}^{2} \mathrm{~min}^{-1}$ and held at a surface pressure of $22 \mathrm{mN} \mathrm{m}^{-1}$, selected to be within the condensed phase of the lipid and to be consistent with previous studies. ${ }^{3,21-23}$ When the lipid film had stabilised, $1 \mathrm{ml}$ of $0.5 \mathrm{mg} \mathrm{ml}^{-1}$ protein solution was added to the sub-phase so that the final concentration of the protein in the trough was $0.48 \mu \mathrm{M}$ respective to either Pin-b or Pin-bs. Experiments were monitored for 150 minutes after protein injection, and changes in surface pressure as a result of protein penetration into the lipid monolayer were plotted as surface pressure vs time. All systems were repeated in triplicate.

Brewster angle microscopy. BAM images were collected using a Nanofilm EP3 imaging ellipsometer (Nanofilm Technology, Goettingen, Germany) mounted above a Langmuir trough (model 302a, Nima, Coventry, UK) so that surface pressure and film morphology could be monitored simultaneously. The imaging ellipsometer was equipped with a frequency doubled $\mathrm{Nd}$ :YaG laser $(532 \mathrm{~nm}, 20 \mathrm{~mW}), 10 \times$ magnification objective, polarizer, analyser and CCD camera. The imaging ellipsomter and film balance were both placed upon an anti-vibration Table. At the Brewster angle nanosized films can be observed on a surface by the reflection of ppolarised light due to small changes in refractive index. Lipid monolayers and protein addition were made as described for surface pressure experiments. The mixed lipid systems composed of 3:2 PE:PG was compared to surface morphology of single phospholipid species and E.coli total lipid extract (Avanti, Madison, WI), the effect of the lipid-protein interaction with respect to Pinb and Pin-bs was then compared using the same lipid systems. The surface pressure was monitored for 180 minutes after protein addition, BAM images were captured at intervals 1, 3, 5, 
$10,15,30,45,60,90,120,150$ and 180 minutes after the protein addition to the sub-phase to monitor surface morphology. Images were taken using an x10 objective lens.

External reflection FTIR Spectroscopy. ER-FTIR spectra were recorded using a Thermo Nicolet Nexus instrument fitted with a monolayer/grazing angle accessory used in conjunction with a $10 \mathrm{ml}$ trough, of which the details of the procedure can be found elsewhere. ${ }^{3,21} \mathrm{In}$ each experiment, $9.5 \mathrm{ml}$ of $20 \mathrm{mM}$ Sodium Phosphate buffer (pD 7.0) was placed in the trough and a background single beam spectra was recorded allowing time for the sample chamber purge to remove $\mathrm{H}_{2} \mathrm{O}$ vapour and $\mathrm{CO}_{2}$ from the atmosphere. After recording a background, $3 \mu 1$ of $1 \mathrm{mg}$ $\mathrm{ml}^{-1}$ lipid solution was spread on to the surface of the buffer and compressed to $22 \mathrm{mN} \mathrm{m}^{-1}$ in the condensed phase. The positions were predefined using surface pressure measurements. Sample scans were taken after compression to ensure stability of the lipid film, which was monitored through the observation of the $\mathrm{CH}_{2}$ symmetric and asymmetric stretching frequencies in the phospholipid tails shown in the regions $2854-2850 \mathrm{~cm}^{-1}$ and $2924-2916 \mathrm{~cm}^{-1}$ respectively. The $\mathrm{C}=\mathrm{O}$ region of the head group is monitored in the region $1760-1700 \mathrm{~cm}^{-1}$. Protein solution $(0.6$ $\mathrm{ml}$ of $0.1 \mathrm{mg} \mathrm{ml}^{-1}$ protein solution) was injected into the sub phase in sequential experiments to make a final protein concentration respective to Pin-b or Pin-bs of $0.48 \mathrm{uM}$, Spectra were continuously collected from the moment of protein injection for 15 minutes followed by a single spectrum collection every 15 minutes, experiments were monitored for 180 minutes after protein injection. The interaction of the protein with the lipid monolayer was observed by monitoring the Amide I region, $1700-1600 \mathrm{~cm}^{-1}$ and the aforementioned $\mathrm{CH}_{2}$ asymmetric and symmetric stretching frequencies. 
To correct for any water vapour present $\mathrm{H}_{2} \mathrm{O}$ and $\mathrm{HOD}$ spectra were scaled and subtracted against protein adsorbed spectrum, the degree of subtraction was dependent on the adsorption time as well as the amount of H/D exchange. The HOD spectra used for scaling and subtraction purposes were collected during the purge of the sample area between single beam background collection and the addition of the lipid film. No further processing was performed to the data.

\section{RESULTS}

Characterisation of lipid monolayers. Pressure-area isotherms were collected for each of the saturated phospholipids, DPPE and DPPG and DPPE:DPPG mixed films for $0 \%, 20 \%, 40 \%$, 60\%, 80\% and 100\% DPPG (Figure 2(a)). Pressure-area isotherms for unsaturated DOPG 18:1 and E.coli total lipid extract (TLE) are given in Figure 2(b). Brewster angle microscopy images were collected concurrently and selected data is shown in Figure 3.

The pressure-area isotherm of DPPG displays the characteristic liquid extended-liquid condensed phase transition during compression to the condensed phase, which was confirmed through observation of progressive domain formation recorded in BAM images as shown in Figure 3(a). In contrast pressure-area isotherms of DPPE exhibited condensed film behaviour, with a sharp transition from the gaseous phase straight into the condensed phase. Supporting this observation, corresponding BAM data in Figure 3(b) showed the spontaneous formation of condensed domains.

When DPPE and DPPG are mixed together, the pressure-area isotherm for the 4:1 PG:PE film showed a complete removal of the phase features that are seen for $100 \%$ DPPG. On increasing 
the percentage of DPPE, the pressure-area isotherms became sharper and at 2:3 PG:PE the pressure-area isotherm resembled that of the 100\% DPPE film. BAM images of the 2:3 PG:PE system show similarities when comparing the two lipid systems, but the 2:3 PG:PE system appears to differ from $100 \%$ DPPE at low compression, showing evidence of phase separation (Figure 3(c)). Pressure-area isotherms for unsaturated DOPG 18:1 and E.coli total lipid extract (TLE) both exhibited gaseous film behaviour and no domain or structural features observed in BAM images (Figure 3(d) shows the images for the total lipid extract sample). FTIR data showed that the lipid C-H stretch peak areas were reduced for unsaturated lipid systems
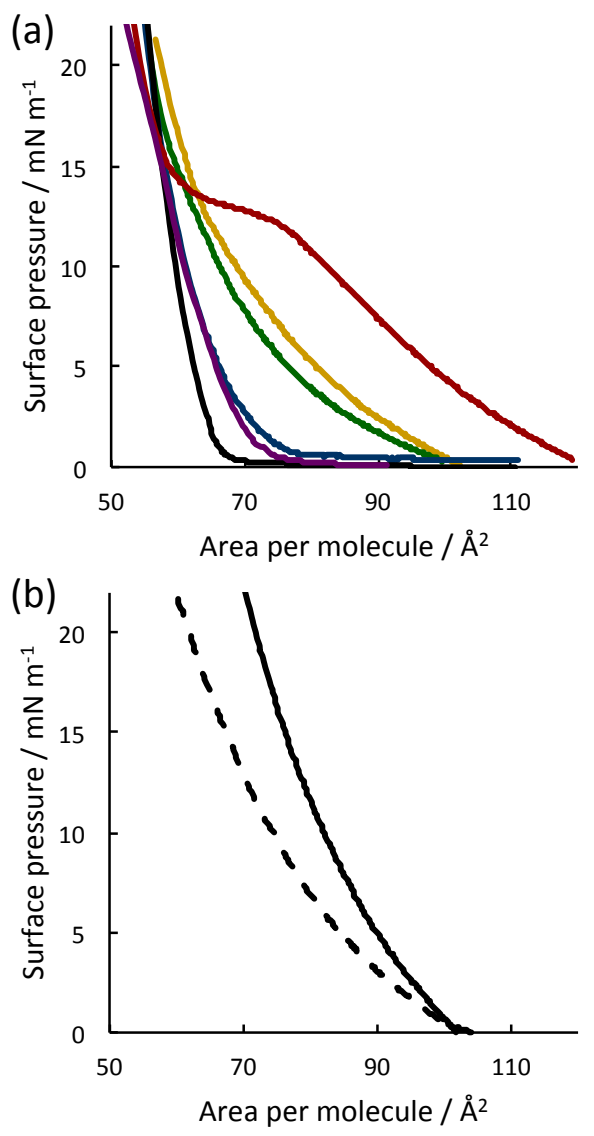

compared to the saturated lipids. This suggested less ordered and a less dense lipid layer when the lipid tails are unsaturated. ${ }^{11}$ The spectra are shown in supplementary material (Figure S1). 
Figure 2. (a) Surface pressure against area per molecule of phospholipid monolayers: $100 \%$ DPPG (red), 4:1 DPPG:DPPE (yellow), 3:2 DPPG:DPPE (green), 2:3 DPPG:DPPE (blue), 1:4 DPPG:DPPE (purple) and 100\% DPPE (black). (b) Surface pressure against area per molecule compression isotherms for the unsaturated DOPG 18:1 (solid) and E.coli total lipid extract (dashed).

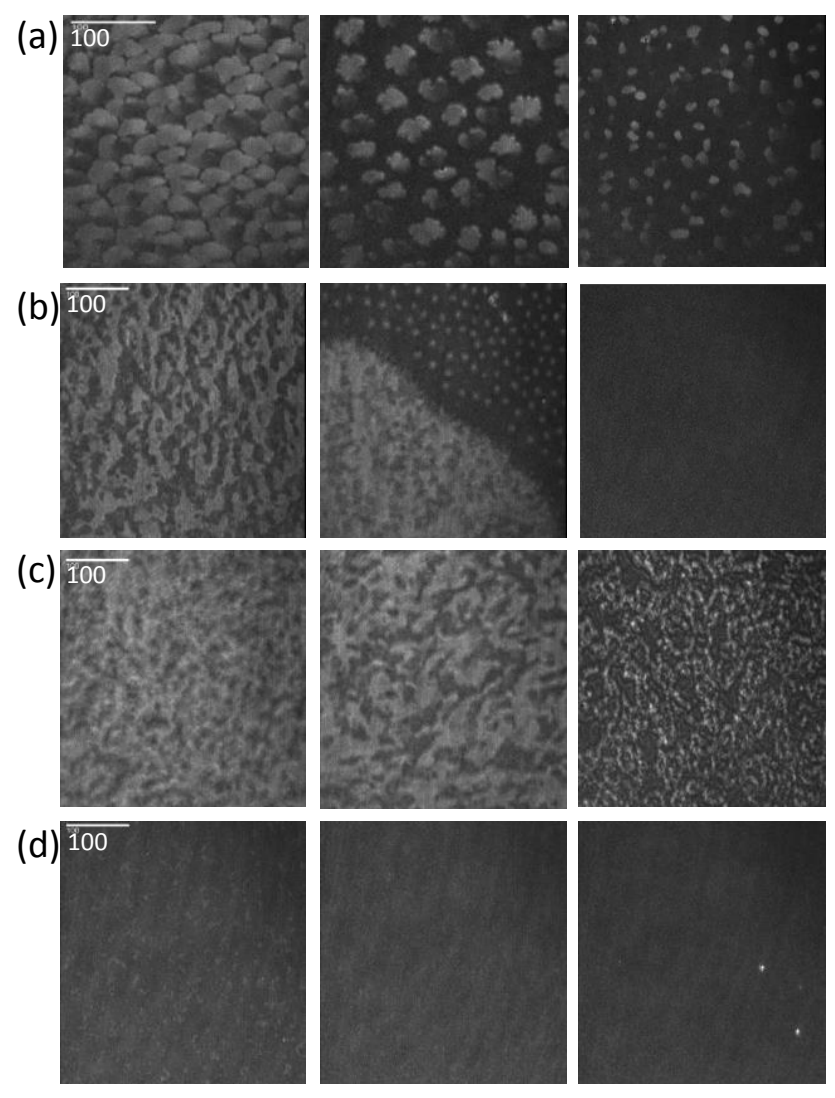

Figure 3. Brewster angle microscopy images taken during monolayer compression of a surface pressure-area isotherm for (a) DPPG only (b) DPPE only (c) 2:3 DPPG:DPPE and (d) E.coli TLE. For each sample the right image was taken at low compression during the gaseous phase ( 0 $\mathrm{mN} \mathrm{m}^{-1}$ ), the center image was taken during mid compression phase transition (approximately 
12-13 $\mathrm{mN} \mathrm{m}^{-1}$ ) and the left image shows the image of the compressed film in its condensed phase at approximately $22 \mathrm{mN} \mathrm{m}^{-1}$ (see surface pressure-area isotherms in Figure 2).

Puroindoline interaction with condensed phase DPPE:DPPG monolayers. The impact that addition of Pin-b and Pin-bs had on mixed layers of DPPG and DPPE from 100\% DPPG to $100 \%$ DPPE was measured by the change in surface pressure and, using ER-FTIR, by the change in peak area of the protein amide $\mathrm{I}$ band $\left(1650 \mathrm{~cm}^{-1}\right)$. Data is summarized in Figure 4 and values given in Table 1. Changes in surface pressure are known to be predominantly sensitive to protein penetration into the lipid layer rather than a measure of total amount of protein at the interface. ${ }^{21-}$ ${ }^{22}$ Upon Pin-b addition to a DPPG monolayer the equilibrium surface pressure change was $10.4 \pm$ $0.6 \mathrm{mN} \mathrm{m}^{-1}$ closely agreeing with previously published results by Clifton et al. ${ }^{3,23}$ Pin-b addition to a DPPE monolayer resulted in an equilibrium surface pressure change of $7.9 \pm 0.7 \mathrm{mN} \mathrm{m}^{-1}$. Interestingly, this value is larger than that observed for Pin-b adsorption to the zwitterionic DPPC $\left(4.3 \pm 0.1 \mathrm{mN} \mathrm{m}^{-1}\right)$ seen in our previous experiments, indicating that head group size may also play an important role. ${ }^{24}$ For Pin-bs, adsorption to a DPPG monolayer led to a change in surface pressure of $7.5 \pm 0.9 \mathrm{mN} \mathrm{m}^{-1}$ in line with previous studies. ${ }^{3,23}$ Pin-bs addition to a DPPE monolayer led to a surface pressure change of only $0.7 \pm 0.7 \mathrm{mN} \mathrm{m}^{-1}$, suggesting little to no penetration of protein into the lipid film. Again, this result differs considerably to that seen previously for Pin-bs binding to DPPC $\left(5.5 \pm 0.4 \mathrm{mN} \mathrm{m}^{-1}\right)$ as further evidence that the interaction depends on factors beyond head group charge. Protein adsorption to mixed DPPE:DPPG systems showed that the change in surface pressure gradually decreased upon increasing zwitterionic DPPE content of the lipid layer. The trend was more pronounced for Pin-bs compared to the Pinb wild type. 
The Amide I peak area from ER-FTIR spectroscopy data provided a measure of total protein at the surface irrespective of level of penetration into the lipid film. Figure 4(b) shows that the equilibrium value of the amide I peak area decreased gradually as the amount of DPPE within the monolayer increased. At 100\% DPPE there was no detectable Amine I peak on addition of either protein to the lipid. For systems with higher DPPG content, the mutant Pin-bs adsorption led to higher peak area values compared to the wild type Pin-b until the amount of DPPE within the monolayer reached $80 \%$. The amide I peak shape was symmetrical highlighting helical nature of the protein for binding to pure and mixed lipid systems (example data shown in supplementary information, Figure S2).

The difference in trends observed between ER-FTIR amide I peak areas and changes in surface pressure upon addition of protein to the mixed lipid layers signify similarities between proteins in terms of amount adsorbed to the surface, but differences in terms of degree of penetration into the lipid film. Pin-b is more effective at penetrating lipid films with high DPPE content compared to Pin-bs. 

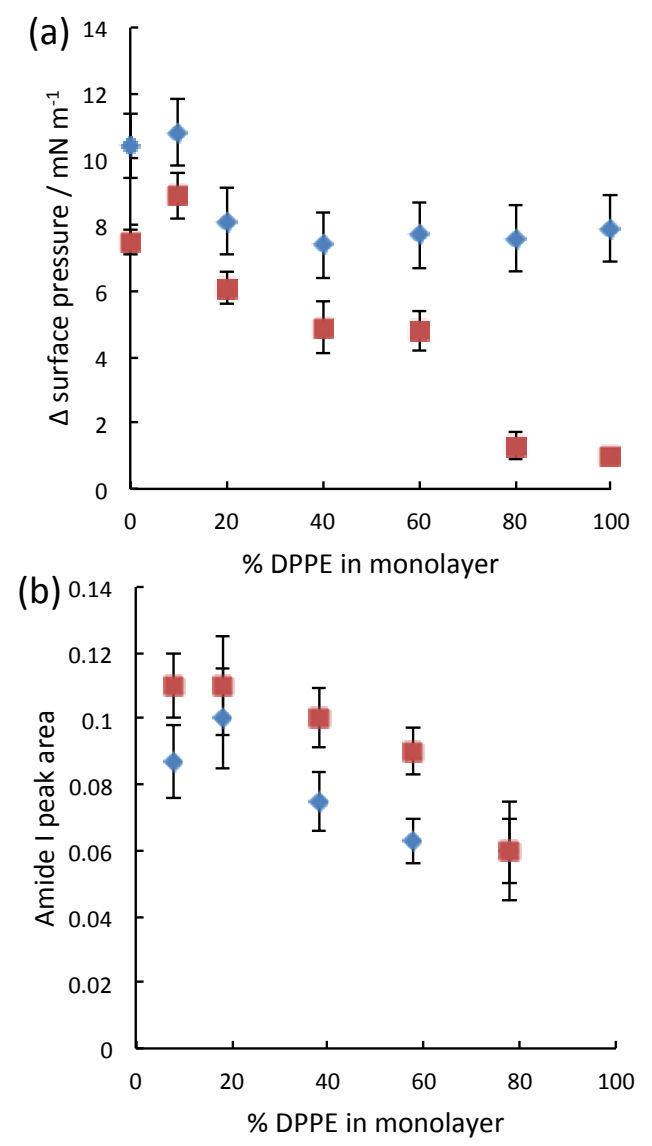

Figure 4. Summary of changes in surface pressure (a) and Amide I peak area (b) upon the addition of Pin-b (blue diamonds) and Pin-bs (red squares) to binary phospholipid monolayers with increasing percentage of DPPE relative to the amount of DPPG within the monolayer. 
Table 1. Change in surface pressure $(\Delta \pi)$ and Amide I peak area for addition of Pin-b and the mutant Pin-bs to binary lipid monolayers consisting of DPPG and DPPE $(n=3)$.

\begin{tabular}{ccccc}
\hline & \multicolumn{2}{c}{ Pin-b } & \multicolumn{2}{c}{ Pin-bs } \\
\hline $\begin{array}{c}\text { Binary } \\
\text { phospholipid } \\
\text { ratio }\end{array}$ & $\Delta \pi / \mathbf{~ m N ~ m}^{-1}$ & $\begin{array}{c}\text { Amide I peak } \\
\text { area }\end{array}$ & $\Delta \pi / \mathbf{~ N ~ N ~ m}^{-1}$ & $\begin{array}{c}\text { Amide I peak } \\
\text { area }\end{array}$ \\
\hline $100 \%$ PG & $10.4 \pm 0.6$ & $0.080 \pm 0.012$ & $7.5 \pm 0.9$ & $0.11 \pm 0.011$ \\
9:1 PG:PE & $10.8 \pm 0.5$ & $0.087 \pm 0.011$ & $8.9 \pm 0.7$ & $0.11 \pm 0.013$ \\
4:1 PG:PE & $8.1 \pm 0.8$ & $0.110 \pm 0.015$ & $6.1 \pm 0.5$ & $0.11 \pm 0.015$ \\
$3: 2$ PG:PE & $7.4 \pm 0.6$ & $0.075 \pm 0.009$ & $4.9 \pm 0.8$ & $0.10 \pm 0.00$ \\
2:3 PG:PE & $7.2 \pm 0.5$ & $0.063 \pm 0.007$ & $4.8 \pm 0.8$ & $0.096 \pm 0.007$ \\
$1: 4$ PG:PE & $6.9 \pm 0.8$ & $0.061 \pm 0.010$ & $1.3 \pm 0.4$ & $0.06 \pm 0.015$ \\
$100 \%$ PE & $7.9 \pm 0.7$ & N/A & $0.7 \pm 0.7$ & N/A \\
\hline
\end{tabular}

The 2:3 PG:PE lipid system was further analysed using BAM (Figures 5 and 6), since this lipid composition closely matched the head group charge composition found in E.coli total lipid extract. Figure 5 shows images taken using BAM as well as surface pressure and the ER-FTIR data for the addition of Pin-b to a the 2:3 PG:PE lipid surface, and Figure 6 provides the corresponding data for Pin-bs. Upon examination of the Pin-b data, BAM images (Figure 5(a)) showed an increase in the brightness of the image upon the addition of the protein compared to the lipid monolayer alone. This brightness change was as a result of an increase in layer thickness implying a large amount of uniform protein adsorbed at the lipid interface. 
Due to the brightness of the Pin-b-lipid image, the laser power was lowered to enable the surface morphology to be determined (large BAM image of Figure 5). In this image a number of small dark spots were observed as a result of defects forming within the monolayer that were not present before the addition of Pin-b but observed previously for Pin-b binding to a DPPG layer. ${ }^{23}$ However, the ER-FTIR data showed no measurable change in the peak area of the $\mathrm{CH}_{2}$ asymmetric stretch and thus the defects seen from BAM images do not appear to be a result of loss of lipid from the monolayer.
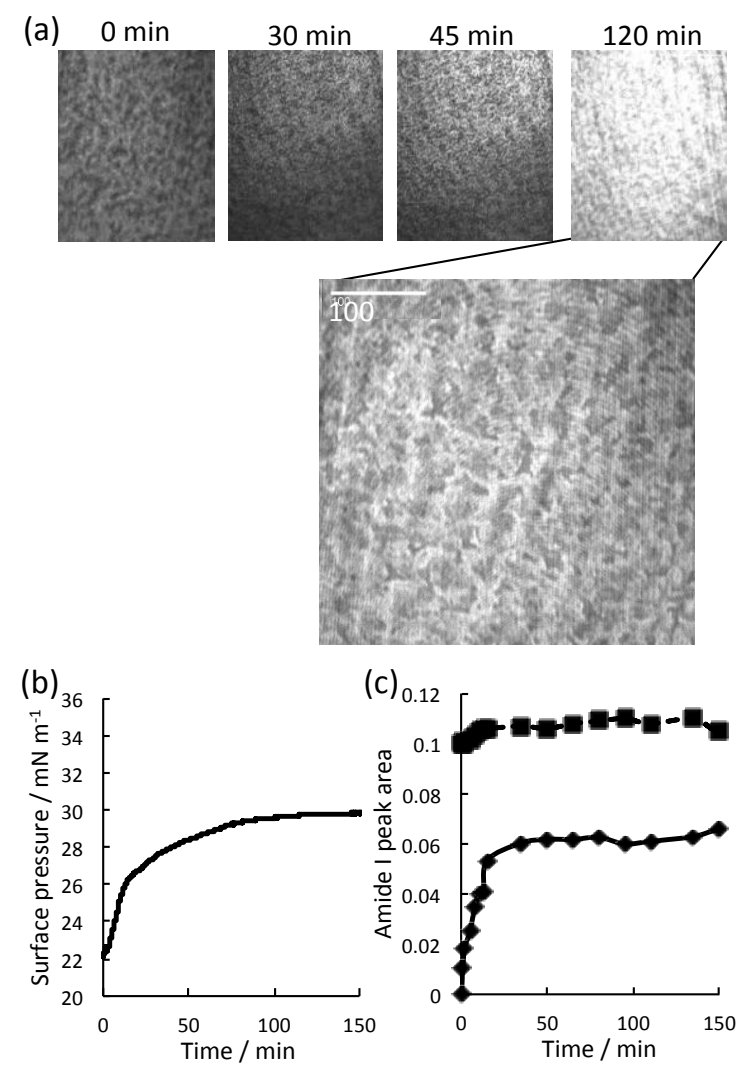

Figure 5. Comparative data for the interaction of Pin-b to 2:3 PG:PE. BAM images (Part (a)) linking to time points of the Surface pressure against time curve (Part (b)) and an inset image showing morphology changes at $120 \mathrm{~min}$ at a lower laser power. Amide I peak area against time (Part (c)) shows changes in Amide I peak area in response to protein adsorption (diamonds) and 
peak area of the $\mathrm{CH}_{2}$ asymmetric stretch (squares). Protein addition to liquid subphase of lipid monolayer at $\mathrm{t}=0$.

For the adsorption of Pin-bs to a 2:3 PG:PE monolayer, BAM images showed that over time there was a brightness change that suggested an increase in thickness of the interfacial material (Figure 6). In contrast to that seen for Pin-b, the change in brightness started to occur within specific domains before a uniform brightness throughout the image. The adsorption of Pin-bs to the 2:3 PG:PE lipid layer was also observed as an increase in the peak area of the Amide I by ER-FTIR and this showed more adsorption of the protein compared to that observed for Pin-b (peak area of $0.096 \pm 0.007$ compared to $0.063 \pm 0.007$ for Pin-b). There was, however, only a modest increase in surface pressure for Pin-bs binding to the lipid surface at $4.8 \mathrm{mN} \mathrm{m}^{-1}$.
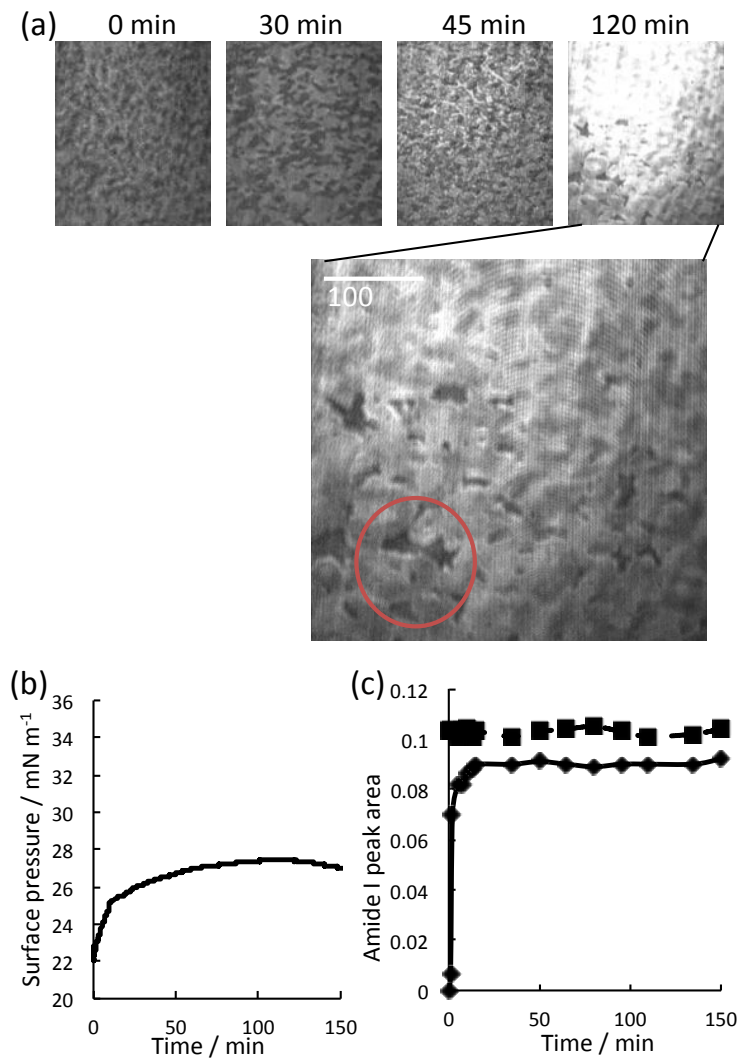
Figure 6. Comparative data for the interaction of Pin-bs to 2:3 PG:PE E.coli mimetic monolayer. BAM images (a) show surface adsorption over time with (inset) showing distinct morphology changes on lower laser power. Time vs surface pressure (b) isotherm of protein penetration displays protein penetration and peak area vs time (c) shows changes in Amide I peak area in response to protein adsorption (diamonds) and any significant changes to the phase of the lipid monolayer (squares).

\section{Puroindoline interaction with E.coli total lipid extract and unsaturated lipid layers.}

Figure 7 shows the BAM, ER-FTIR and surface pressure measurements for Pin-b and Pin-bs interaction with a E.coli total lipid extract (TLE) monolayer. BAM images of Pin-b adsorption showed evidence of protein adsorption after 45 minutes through the formation of bright spots that suggested areas of protein accumulation. Over time there was an increase in uniform brightness at the interface and specific dense areas of brightness, or protein, that resembled "cracks" appearing in the surface morphology. The BAM images for Pin bs adsorption to E.coli TLE were similar showing evidence of uniform adsorption with a uniform increase in brightness after 45 min adsorption.

The increase in surface pressure during adsorption was $13.1 \pm 1.5 \mathrm{mN} \mathrm{m}^{-1}$ for Pin $\mathrm{b}$ and $9.0 \pm$ $0.7 \mathrm{mN} \mathrm{m}^{-1}$ for Pin bs, values that are $40 \%$ and $47 \%$ greater than that seen for their respective binding to 2:3 PG:PE lipid layer. This would suggest that the level of penetration of protein into the lipid layer was higher for the E.coli system. The change in the amide I peak area for both Pin $\mathrm{b}$ and Pin bs was similar in magnitude to that observed for the 2:3 PG:PE lipid system, which shows that the total amount of protein adsorption to the interface is the same or similar to both lipid systems. The total charge ratio between these two lipid systems is believed to be similar, 
however the E.coli TLE layer contains both saturated and unsaturated lipid and will therefore be more fluid than the saturated 2:3 PE:PG lipid systems studied.

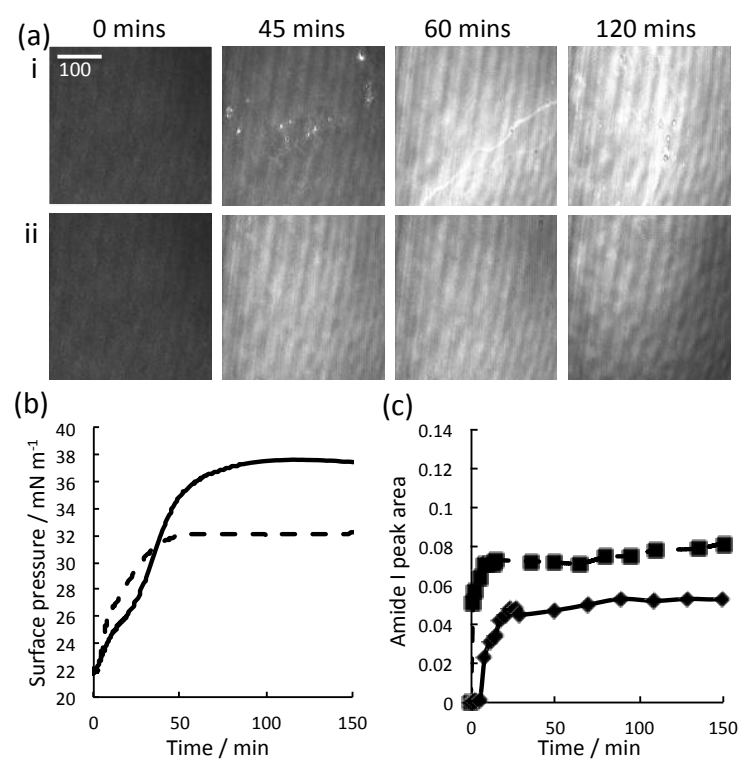

Figure 7. The interaction of Pin-b and Pin-bs to an E.coli TLE monolayer. BAM images (a) show surface adsorption over time of Pin-b (i) and Pin-bs (ii) to the interface. Time vs surface pressure (b) isotherm of protein penetration displays protein penetration of Pin-b (solid) and Pinbs (dashed) and peak area vs time (c) shows changes in Amide I peak area in response to adsorption of Pin-b (diamonds) and Pin-bs (squares).

Table 2. Changes in surface pressure and Amide I peak area upon the addition of Pin-b and Pinbs to lipid systems containing unsaturated phospholipids $(\mathrm{n}=3)$

\section{Pin-b}

Pin-bs

\section{Phospholipid System $\Delta \pi / \mathrm{mN} \mathrm{m}^{-1}$ Amide I peak area $\Delta \pi / \mathrm{mN} \mathrm{m}^{-1}$ Amide I peak area}




\begin{tabular}{ccccc}
\hline E.coli TLE & $13.1 \pm 1.5$ & $0.062 \pm 0.012$ & $9.0 \pm 0.7$ & $0.085 \pm 0.008$ \\
DPPG 16:0 & $10.4 \pm 0.6$ & $0.080 \pm 0.012$ & $7.5 \pm 0.9$ & $0.11 \pm 0.011$ \\
DOPG 18:1 & $11.7 \pm 0.5$ & $0.122 \pm 0.014$ & $11.3 \pm 1.5$ & $0.095 \pm 0.012$ \\
DPPE 16:0 & $7.9 \pm 0.7$ & - & $0.7 \pm 0.7$ & - \\
DPPE 16:1 & $9.8 \pm 1.2$ & - & $5.5 \pm 0.9$ & - \\
\hline
\end{tabular}

To investigate the role lipid chain saturation has on the protein interaction, surface pressure and ER-FTIR experiments were carried out for protein binding to simple unsaturated DOPG 18:1 and DPPE 16:1 lipid layers. The resulting change in surface pressure and Amide I peak areas are given in Table 2 (figures also provided as supplementary material, Figure S3). On comparison between protein binding to DOPG 18:1 and to DPPG it can be seen that the surface pressure change for Pin-b was similar for both lipid systems. In contrast Pin-bs was able to penetrate more into a layer composed of DOPG 18:1 compared to DPPG. When comparing peak areas after 150 min for the unsaturated DOPG 18:1 and the saturated DPPG, a $53 \%$ increase of the Amide I peak area $(0.122 \pm 0.014$ compared to $0.080 \pm 0.012)$ was observed for the interaction of Pin-b suggesting more protein located at the unsaturated lipid interface. In contrast, the peak area data for Pin-bs was similar for both lipid surfaces $(0.095 \pm 0.012$ compared to $0.110 \pm 0.011)$, which suggested that there were similar amounts of protein present at these interfaces. The differences between the proteins observed from surface pressure and FTIR data suggests that the fluidity of the membrane architecture impacted on protein-lipid binding behaviour in terms of protein penetration into the layer for Pin-bs. However, for Pin-b increased adsorption to the surface was observed for the unsaturated lipid but with no obvious increase in surface pressure compared to the saturated lipid. This is likely to be because the surface pressure had reached a maximum for both lipid surfaces. 
The impact of lipid unsaturation on a zwitterionic lipid system was investigated through studying Pin-b and Pin-bs binding to DPPE 16:1 and data shown in Table 2. For Pin-b a 25\% increase in surface pressure change was observed when comparing the saturated and unsaturated DPPE data $\left(7.9 \pm 0.7\right.$ compared $\mathrm{mN} \mathrm{m}^{-1}$ to $\left.9.8 \pm 1.2 \mathrm{mN} \mathrm{m}^{-1}\right)$. For Pin-bs binding there was a more dramatic increase when the lipid was unsaturated, with the change in surface pressure rising from $0.7 \pm 0.7 \mathrm{mN} \mathrm{m}^{-1}$ to $5.5 \pm 0.9 \mathrm{mN} \mathrm{m}^{-1}$. Both these observations indicated increased protein penetration into the unsaturated layer compared to the saturated lipid.

\section{DISCUSSION}

In this study we have used mixed phospholipid monolayers at the air/liquid interface to demonstrate the effect of head group electrostatics and acyl tail saturation on protein-lipid interactions for wild type Pin-b and its mutant Pin-bs. Results from compression isotherms of single lipids DPPE and DPPG agree well with previously published data. ${ }^{3,23,25-27}$ Pressure-area isotherms of the binary phospholipid mixtures show evidence of phase separation of the two phospholipids agreeing with BAM images collected in both the gaseous and the condensed phase. This also agrees with BAM experiments using PE/PG mixed compositions seen in the literature. ${ }^{27}$ Pressure-area isotherms collected that contained unsaturated lipids, such as the E.coli TLE, did not display any obvious domain structure and this was confirmed from BAM images taken of E.coli TLE lateral structure at the interface.

The methods used here are sensitive to two different types of protein-lipid interaction, penetration into the layer and thus disruption of the lipid organisation, as measured by BAM and surface pressure changes, and total amount (mass) of protein at the interface that is proportional 
to the peak area of the FTIR amide I peak. For Pin binding to the PG-PE systems we find that strong adsorption to the lipid surface measured by the size of the FTIR amide I peak does not necessarily mean a corresponding large change in surface pressure, because bound protein does not always penetrate or disrupt the lipid tail region. ${ }^{22}$ However, we would expect that the ability of the protein to disrupt/penetrate the lipid layer would link more closely to cell lysis activity compared to weaker interactions with the lipid head group only. ${ }^{18,28}$ Our results show that as we gradually increase the content of PE within the lipid layer the amount of protein adsorption decreases until levels are not detectable by ER-FTIR at 100\% PE. This is true for both Pin-b and Pin-bs and so in terms of adsorption to the surface both proteins behave similarly. However, BAM and surface pressure measurements show differences in terms of lipid penetration with the wild type Pin-b more able to penetrate PE containing lipid layers compared to Pin-bs. This shows that the loss of the third hydrophobic Trp residue impacts on the ability of Pin-bs to penetrate into the lipid tail region of the lipid layer. This is compounded by the fact that the Trp is replaced by a cationic Arg group in Pin-bs, promoting association with the head group region of the lipids rather than deeper penetration.

The lipid charge distribution in E.coli lipid extract is approximately a 2:3 mix of anionic:zwitterionic lipid, primarily made up of PE, PG and cardiolipin. ${ }^{12}$ Our analysis of a 2:3 PG:PE lipid layer shows a build up of protein below the lipid layer and evidence of phase changes to the lipid layer. Pin-b is shown by surface pressure measurements to penetrate the lipid layer more effectively than Pin-bs, showing a surface pressure increase that is $50 \%$ greater. However, according to our FTIR measurements the total amount of protein at the lipid surface is 34\% less for Pin-b than Pin-bs. BAM images show some evidence of lipid disruption in the presence of both proteins; however for Pin-bs the dark features seem larger and less widespread. 
These results can be explained when considering the single point mutation of Pin-bs. Pin-bs has additional charge within the Trp rich loop of the protein, which would allow for more adsorption to the surface and head group of the lipid. The additional Trp of Pin-b wild type would promote deeper penetration. ${ }^{3}$ In terms of activity of the proteins, it is reasonable to expect pore formation for Pin-bs after the lipid surface is covered with adsorbed protein. Dark features are observed by BAM that may represent this mechanism. The Pin-bs loop has distinct hydrophilic and hydrophobic regions, with the amino acids near the cysteine bond more hydrophilic than those within the centre of the loop, thus making the loop amphiphilic in nature and limiting depth of penetration into the lipid hydrophobic region (see Figures 8). The difference made by the Trp instead of Arg in the wild type Pin-b is to render one side of the loop predominantly hydrophobic thus enhancing penetration of the loop within the hydrophobic region of the lipid layer.
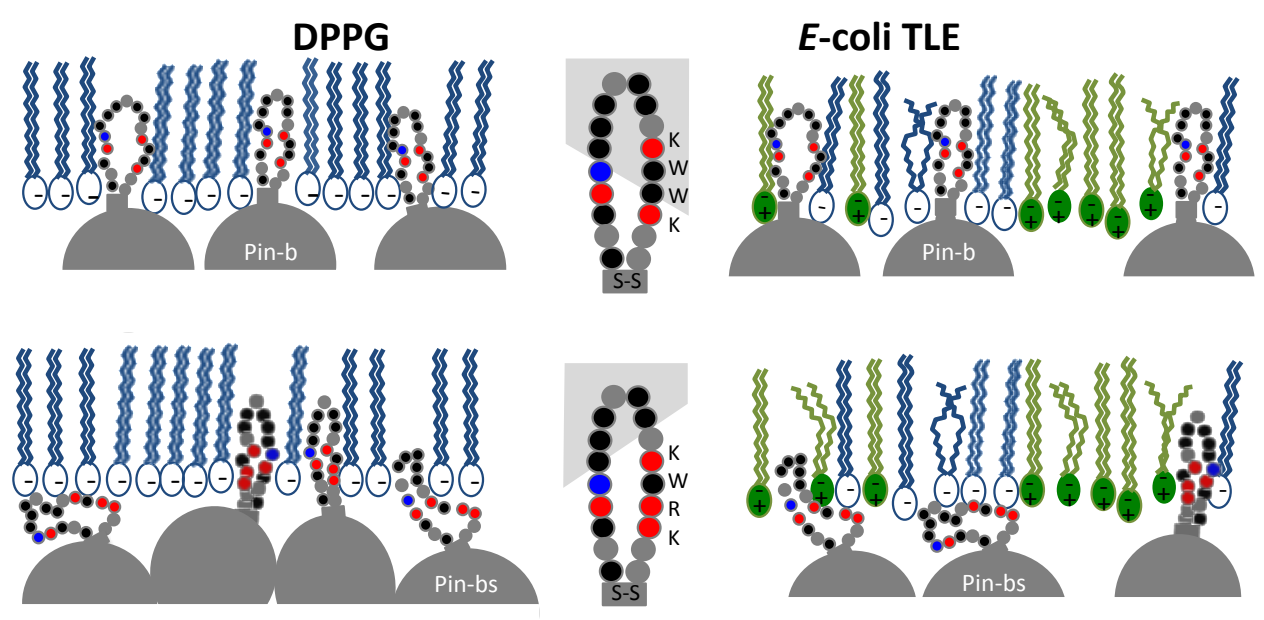

Figure 8. A schematic representation demonstrating the difference in binding of Pin-b and Pinbs to an anionic (DPPG) lipid surface and a E.coli TLE surface. The Trp to Arg substitution in 
Pin-bs changes the dynamics of the hydrophobic lipid-binding loop to an amphiphilic loop with a greater hydrophilic component. This limits the proteins depth of penetration into the lipid tails.

We have asked ourselves the question of how well do our model lipid systems compare to 'real' biological lipid compositions and answered this by investigating Pin binding to monolayers of $E$. coli total lipid extract. The results show that Pins interact more to the E.coli TLE compared to the model 2:3 PG:PE lipid layer and this is likely to be due to the increased fluidity of the heterogeneous lipid layer formed by E. coli TLE. The E.coli TLE does not form domain structures at the surface as seen by BAM. For both proteins the amount of protein interacting with the lipid head group does not show a significant difference between these two lipid systems. Conversely the level of penetration, as seen from surface pressure measurements, doubles on binding to the E.coli TLE compared to 2:3 PG:PE. To investigate the impact of the increased fluidity of the lipid layer, we investigated Pin binding to two unsaturated lipid layers.

On comparing protein binding to unsaturated and saturated lipids for PG (DPPG and POPG 18:1) we observed that there was strong penetration and adsorption of protein to both lipid layers. Most notable is the increase in penetration (or change in surface pressure) of both Pins to the PE 16:1 layer compared to PE 16:0. This observation is particularly apparent for Pin-bs, where the surface pressure change was $0.7 \pm 0.7 \mathrm{mN} \mathrm{m}^{-1}$ for binding the saturated lipid to $5.5 \pm 0.9$ $\mathrm{mN} \mathrm{m}^{-1}$ for binding to the unsaturated lipid. This result confirms that the presence of unsaturated tails provides a lipid surface that is more prone to attack by these proteins. The selectivity and difference in behaviour of the two proteins is still observed but becomes less pronounced. 
In conclusion, this work has shown that the interaction of the Pins with lipid membranes is not solely driven by electrostatic forces. The interaction is also influenced by domain formation of the membrane, which is in turn regulated by head group composition and the presence of unsaturated acyl tails within the lipid acyl region. Furthermore the Trp to Arg substitution within the tryptophan-rich domain of Pin-bs has a strong effect on the interaction that is particularly evident when the complexity of the lipid system is increased. This provokes questions with respect to how the behavior of Pin-bs, and hence the conservation of mutation, is advantageous to the wheat grain. The work provides insight in terms of the role of lipid composition within biological membranes, and shows how subtle differences enhance or inhibit antimicrobial protein or peptide interaction.

\section{ASSOCIATED CONTENT}

\section{Supporting Information.}

Figure S1: ER-FTIR spectra of the C-H stretch region of the lipid layers.

Figure S2: Deconvolution of amide I peak for Pin $b$ and Pin bs after adsorption to DPPG and 3:2 DPPE:DPPG is provided.

Figure S3: Figure showing amide I peak area/surface pressure versus time for Pin interaction with DOPG 18:1 and DPPE 16:1. Data summarized in Table 2

This supporting information is available free of charge as a pdf file on the ACS publications website at http://pubs.acs.org. 


\title{
AUTHOR INFORMATION
}

\section{Corresponding Authors}

*rebecca.green@reading.ac.uk

*r.a.frazier@reading.ac.uk

\section{Notes}

The authors declare no competing financial interest.

\section{ACKNOWLEDGMENT}

This work was financially supported by the UK Science and Technologies Facilities Council and University of Reading.

\author{
ABBREVIATIONS \\ DPPC, 1,2-dipalmitoyl-sn-glycero-3-phosphocholine; DPPG, 1,2-dipalmitoyl-sn-glycero-3- \\ phospho-(1'-rac-glycerol); DPPE, 1,2-dipalmitoyl-sn-glycero-3-phosphoethanolamine, DOPE, \\ 1,2-di-(9Z-octadecenoyl)-sn-glycero-3-phospho-(1'-rac-glycerol); ER-FTIR, External reflection \\ Fourier transform infrared; BAM, Brewster angle microscopy; Pin, puroindoline; UHQ, ultra- \\ high quality
}

\section{REFERENCES}

(1) Bottier, C.; Géan, J.; Artzner, F.; Desbat, B.; Pézolet, M.; Renault, A.; Marion, D.; Vié, V. Galactosyl headgroup interactions control the molecular packing of wheat lipids in Langmuir films and in hydrated liquid-crystalline mesophases. Biochim. Biophys. Acta Biomembr. 2007, 1768 (6), 1526-1540. 
(2) Haney, E. F.; Petersen, A. P.; Lau, C. K.; Jing, W. G.; Storey, D. G.; Vogel, H. J. Mechanism of action of puroindoline derived tryptophan-rich antimicrobial peptides. Biochim. Biophys. Acta Biomembr. 2013, 1828 (8), 1802-1813.

(3) Clifton, L. A.; Lad, M. D.; Green, R. J.; Frazier, R. A. Single amino acid substitutions in puroindoline-b mutants influence lipid binding properties. Biochem. 2007, 46 (8), 22602266.

(4) Epand, R. M.; Epand, R. F. Lipid domains in bacterial membranes and the action of antimicrobial agents. Biochim. Biophys. Acta Biomembr. 2009, 1788 (1), 289-294.

(5) Fernandes, F.; Coutinho, A.; Prieto, M.; Loura, L. M. S. Electrostatically driven lipidprotein interaction: Answers from FRET. Biochim. Biophys. Acta Biomembr. 2015, 1848 (9), 1837-1848.

(6) Nyholm, T. K. M. Lipid-protein interplay and lateral organization in biomembranes. Chemistry and Physics of Lipids 2015, 189, 48-55.

(7) Cybulski, L. E.; de Mendoza, D. Bilayer Hydrophobic Thickness and Integral Membrane Protein Function. Current Protein \& Peptide Science 2011, 12 (8), 760-766.

(8) Strandberg, E.; Ulrich, A. S. AMPs and OMPs: Is the folding and bilayer insertion of $\beta$ stranded outer membrane proteins governed by the same biophysical principles as for $\alpha$ helical antimicrobial peptides? Biochim. Biophys. Acta Biomembr. 2015, 1848 (9), 19441954. 
(9) Callan-Jones, A.; Sorre, B.; Bassereau, P. Curvature-driven lipid sorting in biomembranes. Cold Spring Harbor perspectives in biology 2011, 3 (2), a004648.

$$
\text { Sanchez, S. A.; Alejandra Tricerri, M.; Ossato, G.; Gratton, E. Lipid packing }
$$
determines protein-membrane interactions: Challenges for apolipoprotein A-I and high density lipoproteins. Biochim. Biophys. Acta Biomembr. 2010, 1798 (7), 1399-1408.

$$
\text { Chan, D. I.; Prenner, E. J.; Vogel, H. J., Tryptophan- and arginine-rich }
$$
antimicrobial peptides: Structures and mechanisms of action. Biochim. Biophys. Acta Biomembr. 2006, 1758 (9), 1184-1202.

$$
\text { Epand, R. M.; Epand, R. F. Bacterial membrane lipids in the action of }
$$
antimicrobial agents. J. Peptide Science 2011, 17 (5), 298-305.

$$
\text { Veld, G. I.; Driessen, A. J.; Konings, W. N. Bacterial solute transport proteins in }
$$
their lipid environment. FEMS microbiology reviews 1993, 12 (4), 293-314. Douliez, J. P.; Michon, T.; Elmorjani, K.; Marion, D., Structure, biological and technological functions of lipid transfer proteins and indolines, the major lipid binding proteins from cereal kernels. J. Cereal Science 2000, 32 (1), 1-20.

$$
\text { Giroux, M. J.; Sripo, T.; Gerhardt, S.; Sherwood, J. Puroindolines: Their role in }
$$
grain hardness and plant defence. Biotechnology \& Genetic Engineering Reviews 2003, 20, 277-290.

$$
\text { Morris, C. F. Puroindolines: the molecular genetic basis of wheat grain hardness. }
$$

Plant Molecular Biology 2002, 48 (5), 633-647. 

applications. Biotechnology Advances 2007, 25 (2), 195-197.

Alfred, R. L.; Palombo, E. A.; Panozzo, J. F.; Bhave, M., The Antimicrobial Domains of Wheat Puroindolines Are Cell-Penetrating Peptides with Possible Intracellular Mechanisms of Action. Plos One 2013, 8 (10).

$$
\text { Day, L.; Bhandari, D. G.; Greenwell, P.; Leonard, S. A.; Schofield, J. D., }
$$
Characterization of wheat puroindoline proteins. Febs Journal 2006, 273 (23), 53585373.

Blochet, J. E.; Chevalier, C.; Forest, E.; Pebaypeyroula, E.; Gautier, M. F.; Joudrier, P.; Pezolet, M.; Marion, D. Complete Amino-Acid sequence of Puroindoline, a new basic and Cystein-rich protein with a unique Tryptophan-Rich domain, isolated from wheat endosperm by Triton X-114 phase partitioning. Febs Letters 1993, 329 (3), 336340.

Lad, M. D.; Birembaut, F.; Clifton, L. A.; Frazier, R. A.; Webster, J. R. P.; Green, R. J. Antimicrobial peptide-lipid binding interactions and binding selectivity. Biophys. J. 2007, 92 (10), 3575-3586. Lad, M. D.; Birembaut, F.; Frazier, R. A.; Green, R. J., Protein-lipid interactions at the air/water interface. Phys. Chem. Chem. Phys. 2005, 7 (19), 3478-3485.

Clifton, L. A.; Green, R. J.; Hughes, A. V.; Frazier, R. A. Interfacial Structure of Wild-Type and Mutant Forms of Puroindoline-b Bound to DPPG Monolayers. J. Phys. Chem. B 2008, 112 (49), 15907-15913. 

Caging of DPPC, DPPE, DPPG, and DPPS Monolayers Caused by Dimethylsulfoxide Observed Using Brewster Angle Microscopy. Langmuir 2010, 26 (24), 18902-18908.

Alexandre, S.; Colé, G.; Coutard, S.; Monnier, C.; Norris, V.; Margolin, W.; Yu, X.; Valleton, J. M., Interaction of FtsZ protein with a DPPE Langmuir film. Colloids and Surfaces B: Biointerfaces 2002, 23 (4), 391-395.

$$
\text { Clifton, L. A.; Sanders, M. R.; Hughes, A. V.; Neylon, C.; Frazier, R. A.; Green, }
$$

R. J., Lipid binding interactions of antimicrobial plant seed defence proteins: puroindoline-a and beta-purothionin. Phys. Chem. Chem. Phys. 2011, 13 (38), 1715317162.

$$
\text { Wydro, P.; Flasiński, M.; Broniatowski, M., Molecular organization of bacterial }
$$
membrane lipids in mixed systems-A comprehensive monolayer study combined with Grazing Incidence X-ray Diffraction and Brewster Angle Microscopy experiments. Biochim. Biophys. Acta Biomembr. 2012, 1818 (7), 1745-1754. Capparelli, R.; Amoroso, M. G.; Palumbo, D.; Iannaccone, M.; Faleri, C.; Cresti, M., Two plant puroindolines colocalize in wheat seed and in vitro synergistically fight against pathogens. Plant Molecular Biology 2005, 58 (6), 857-867. 
Table of Contents Graphic

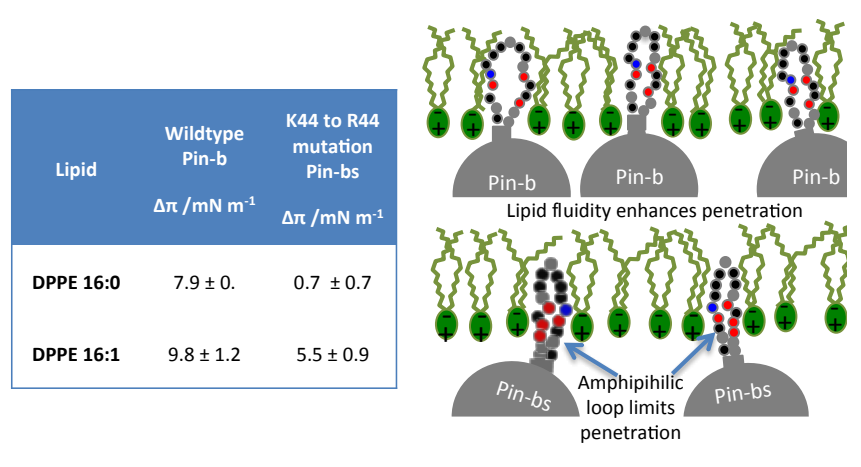

